\title{
An unusual cause of pink diapers in an infant: Questions
}

\author{
Rasheda Amin • Loai Eid • Vidar O. Edvardsson • \\ Lynette Fairbanks • Asha Moudgil
}

Received: 22 January 2015 /Revised: 11 February 2015 / Accepted: 12 February 2015 / Published online: 1 April 2015

(C) IPNA 2015

Keywords Urinary crystals · Pink-stained diapers ·

Hypouricemia $\cdot$ Fractional excretion of uric acid

\section{Case summary}

A 9-month-old, Caucasian, male infant presented for evaluation of intermittent pink diaper staining since the age of 4 months, more often observed in the first morning diapers. He was noted to be straining occasionally while voiding. Additionally, he was not thriving normally, and in the past 5 months his weight had fallen from the 64th to the 8th percentile and length from the 49th to the 37th percentile. There was no history of fever, chronic diarrhea, urinary tract infections or urinary stone passage. He was the product of a full-term gestation and was developmentally normal. He was the first child of nonconsanguineous parents, and the family history was unremarkable. On physical examination, no dysmorphic features were observed and blood pressure was normal; all other findings of the physical examination were within normal limits.

The answers to these questions can be found at http://dx.doi.org/10.1007/ s00467-015-3073-y

R. Amin · A. Moudgil $(\bowtie)$

Division of Pediatric Nephrology, Children's National Health

System, 111 Michigan Avenue NW, Washington, DC 20010, USA

e-mail: amoudgil@childrensnational.org

L. Eid

Division of Pediatrics, Latifa Hospital, Dubai, United Arab Emirates

V. O. Edvardsson

Children's Medical Center, Landspitali-The National University

Hospital of Iceland, Reykjavik, Iceland

V. O. Edvardsson

Faculty of Medicine, School of Health Sciences, University of

Iceland, Reykjavik, Iceland

L. Fairbanks

Purine Research Laboratory, Viapath, St Thomas' Hospital,

London, UK
Initial urinalysis and all laboratory studies, including complete blood count, renal function, liver function, thyroid function, serum magnesium levels, celiac disease panel, venous blood gas, parathyroid hormone and 25-hydroxy-vitamin D levels, were within normal reference ranges. Serum uric acid was 0.1 (reference range 1.4-6.7) $\mathrm{mg} / \mathrm{dl}$. Random urine studies revealed normal urine citrate-, oxalate- and calcium-tocreatinine ratios. Fractional excretion of magnesium and tubular phosphate reabsorption was normal. There was no evidence of generalized aminoaciduria. No abnormalities were visible on a renal and bladder ultrasound, and a radiograph of the wrist was negative for rickets. However, urine microscopic examination of first morning urine revealed the presence of numerous crystals (Fig. 1).

\section{Questions}

1. What are the possible etiologies of crystalluria in an infant?

2. What further testing can be done in this patient to reach a diagnosis?

3. What conditions are associated with hypouricemia?

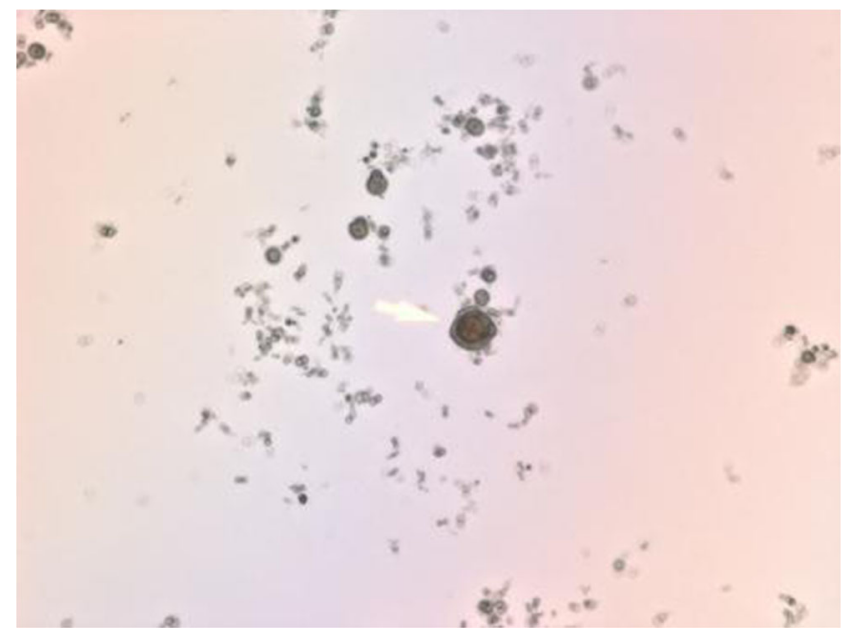

Fig. 1 Urinary crystals $(40 \times)$. Urine microscopy $(40 \times)$ showing a large crystal (arrowhead) with similar, smaller crystals in the background 